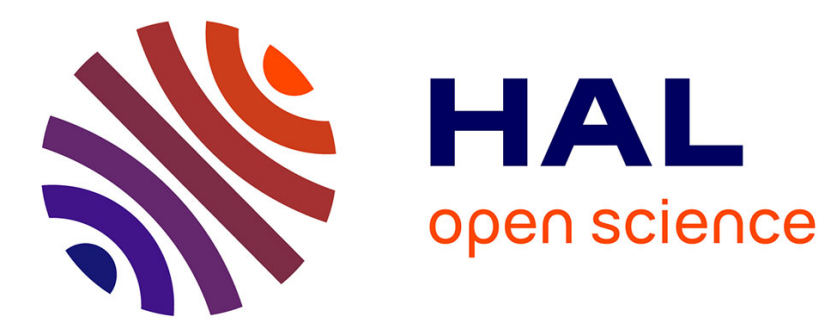

\title{
Implementing fast carryless multiplication
}

Joris van Der Hoeven, Robin Larrieu, Grégoire Lecerf

\section{To cite this version:}

Joris van Der Hoeven, Robin Larrieu, Grégoire Lecerf. Implementing fast carryless multiplication. 2017. hal-01579863

\section{HAL Id: hal-01579863 \\ https://hal.science/hal-01579863}

Preprint submitted on 31 Aug 2017

HAL is a multi-disciplinary open access archive for the deposit and dissemination of scientific research documents, whether they are published or not. The documents may come from teaching and research institutions in France or abroad, or from public or private research centers.
L'archive ouverte pluridisciplinaire HAL, est destinée au dépôt et à la diffusion de documents scientifiques de niveau recherche, publiés ou non, émanant des établissements d'enseignement et de recherche français ou étrangers, des laboratoires publics ou privés. 


\title{
Implementing fast carryless multiplication
}

\author{
JORIS VAN DER HOEVEN ${ }^{a}$, ROBIN LARRIEU ${ }^{b}$, GRÉGOIRE LECERF $^{c}$ \\ Laboratoire d'informatique de l'École polytechnique \\ LIX, UMR 7161 CNRS \\ Campus de l'École polytechnique \\ 1 , rue Honoré d'Estienne d'Orves \\ Bâtiment Alan Turing, CS35003 \\ 91120 Palaiseau, France \\ a. Email: vdhoeven@lix.polytechnique.fr \\ b. Email: larrieu@lix.polytechnique.fr \\ c. Email: lecerf@lix.polytechnique.fr \\ Preliminary version of August 31, 2017
}

\footnotetext{
The efficient multiplication of polynomials over the finite field $\mathbb{F}_{2}$ is a fundamental problem in computer science with several applications to geometric error correcting codes and algebraic crypto-systems. In this paper we report on a new algorithm that leads to a practical speed-up of about two over previously available implementations. Our current implementation assumes a modern AVX2 and CLMUL enabled processor.

KEYWORDS: polynomial multiplication, finite field, Frobenius FFT, implementation, high performance

A.C.M. SUBJECT CLASSIFICATION: G.1.0.0: Computer arithmetic, F.2.1.1: Computations in finite fields, F.2.1.0: Computation of transforms (e.g., fast Fourier transform)

A.M.S. SUBJECT CLASSIFICATION: 68W30, 68Q17, 68W40
}

\section{INTRODUCTION}

Modern algorithms for fast polynomial multiplication are generally based on evaluation-interpolation strategies and more particularly on the discrete Fourier transform (DFT). Taking coefficients in the finite field $\mathbb{F}_{2}$ with two elements, the problem of multiplying in $\mathbb{F}_{2}[x]$ is also known as carryless integer multiplication (assuming binary notation). The aim of this paper is to present a practically efficient solution for large degrees.

One major obstruction to evaluation-interpolation strategies over small finite fields is the potential lack of evaluation points. The customary remedy is to work in suitable extension fields. Remains the question of how to reduce the incurred overhead as much as possible.

More specifically, it was shown in [6] that multiplication in $\mathbb{F}_{2}[x]$ can be done efficiently by reducing it to polynomial multiplication over the Babylonian field $\mathbb{F}_{2^{60}}$. Part of this reduction relied on Kronecker segmentation, which involves an overhead of a factor two. In this paper, we present a variant of a new algorithm from [10] that removes this overhead almost entirely. We also report on our MATHEMAGIX implementation that is roughly twice as efficient as before.

\subsection{Related work}

For a long time, the best known algorithm for carryless integer multiplication was Schönhage's triadic variant [15] of Schönhage-Strassen's algorithm [16] for integer multiplication: it achieves a complexity $O(n \log n \log \log n)$ for the multiplication of two 
polynomials of degree $n$. Recently [7], Harvey, van der Hoeven and Lecerf proved the sharper bound $O\left(n \log n 8^{\log ^{*} n}\right)$, but also showed that several of the new ideas could be used for faster practical implementations [6].

More specifically, they showed how to reduce multiplication in $\mathbb{F}_{2}[x]$ to DFTs over $\mathbb{F}_{260}$, which can be computed efficiently due to the existence of many small prime divisors of $2^{60}-1$. Their reduction relies on Kronecker segmentation: given two input polynomials $A(x)=\sum_{0 \leqslant i<n} a_{i} x^{i}$ and $B(x)=\sum_{0 \leqslant i<n} a_{i} x^{i}$ in $\mathbb{F}_{2}[x]$, one cut them into chunks of 30 bits and form $\tilde{A}(y, z)=\sum_{i=0}^{m-1} \sum_{j=0}^{29} a_{30 i+j} z^{j} y^{i}$ and $\tilde{B}(y, z)=\sum_{i=0}^{m-1} \sum_{j=0}^{29} b_{30 i+j} z^{j} y^{i}$, where $m=\lceil n / 30\rceil$ (the least integer $\geqslant n / 30$ ). Hence $A(x)=\tilde{A}\left(x^{30}, x\right), B(x)=\tilde{B}\left(x^{30}, x\right)$, and the product $C=A B$ satisfies $C(x)=\tilde{C}\left(x^{30}, x\right)$, where $\tilde{C}=\tilde{A} \tilde{B}$. Now $\tilde{A}$ and $\tilde{B}$ are multiplied in $\mathbb{F}_{2^{60}}[x]$ by reinterpreting $z$ as the generator of $\mathbb{F}_{2^{60}}$. The recovery of $\tilde{C}$ is possible since its degree in $z$ is bounded by $2 \cdot 29=58<60$. However, in terms of input size, half of 60 coefficients of $\tilde{A}(y, z)$ and $\tilde{B}(y, z)$ in $z$ are "left blank", when reinterpreted inside $\mathbb{F}_{2^{60}}$. Consequently, this reduction method based on Kronecker segmentation involves a constant overhead of roughly 2 . In fact, when considering algorithms with asymptotically softly linear costs, comparing relative input sizes gives a rough approximation of the relative costs.

Recently van der Hoeven and Larrieu [10] have proposed a new way to reduce multiplication of polynomials in $\mathbb{F}_{q}[x]$ to the computation of DFTs over an extension $\mathbb{F}_{q}$. Roughly speaking, they have shown that the DFT of a polynomial in $\mathbb{F}_{q^{\ell}}[x]$ could be computed almost $\ell$ times faster if its coefficients happen to lie in the subfield $\mathbb{F}_{q}$. Using their algorithm, called the Frobenius FFT, it is theoretically possible to avoid the overhead of Kronecker segmentation, and thereby to gain a factor of two with respect to [6]. However, application of the Frobenius FFT as described in [10] involves computations in all intermediate fields $\mathbb{F}_{q^{e}}$ between $\mathbb{F}_{q}$ and $\mathbb{F}_{q^{\ell}}$. This makes the theoretical speed-up of two harder to achieve and practical implementations more cumbersome.

Besides Schönhage-Strassen type algorithms, let us mention that other strategies such as the additive Fourier transform have been developed for $\mathbb{F}_{2^{k}}[x][3,14]$; but to the best of our knowledge they have not led so far to competitive implementations for large sizes. For more historical details on the complexity of polynomial multiplication we refer the reader to the introductions of $[6,7]$ and to the book by von zur Gathen and Gerhard [4].

\subsection{Results and outline of the paper}

This paper contains two main results. In section 3, we describe a variant of the Frobenius DFT for the special extension of $\mathbb{F}_{2^{60}}$ over $\mathbb{F}_{2}$. Using a single rewriting step, this new algorithm reduces the computation of a Frobenius DFT to the computation of an ordinary DFT over $\mathbb{F}_{2^{60}}$, thereby avoiding computations in any intermediate fields $\mathbb{F}_{2^{e}}$ with $1<e<60$ and $e \mid 60$.

Our second main result is a practical implementation of the new algorithm and our ability to indeed gain a factor that approaches two. In section 4, we present some of the low level implementation details concerning the new rewriting step. Our timings are presented in section 5 . Our implementation outperforms the reference library GF $2 x$ version 1.2 developed by Brent, Gaudry, Thomé and Zimmermann [1] for multiplying polynomials in $\mathbb{F}_{2}[x]$. Finally, the evaluation-interpolation strategy used by our algorithm is particularly well suited for multiplying matrices of polynomials over $\mathbb{F}_{2}$, as reported in section 5 . 


\section{PRereQuisites}

\section{Discrete Fourier transforms}

Let $\omega$ be a primitive root of unity of order $n$ in $\mathbb{F}_{q}$. The discrete Fourier transform (DFT) of an $n$-tuple $a=\left(a_{0}, \ldots, a_{n-1}\right) \in \mathbb{F}_{q}^{n}$ with respect to $\omega$ is $\operatorname{DFT}_{\omega}(a):=\left(\hat{a}_{0}, \ldots, \hat{a}_{n-1}\right) \in \mathbb{F}_{q}^{n}$, where

$$
\hat{a}_{i}:=a_{0}+a_{1} \omega^{i}+\cdots+a_{n-1} \omega^{(n-1) i} .
$$

Hence $\hat{a}_{i}$ is the evaluation of the polynomial $A(x)=a_{0}+a_{1} x+\cdots+a_{n-1} x^{n-1}$ at $\omega^{i}$. For simplicity we often identify $A$ with $a$ and we simply write $\operatorname{DFT}_{\omega}(A)$. The inverse transform is related to the direct transform via $\mathrm{DFT}_{\omega}^{-1}=n^{-1} \mathrm{DFT}_{\omega^{-1}}$, which follows from the well known formula

$$
\operatorname{DFT}_{\omega^{-1}}\left(\operatorname{DFT}_{\omega}(a)\right)=n a .
$$

If $n$ properly factors as $n=n_{1} n_{2}$, then $\omega^{n_{1}}$ is an $n_{2}$-th primitive root of unity and $\omega^{n_{2}}$ is an $n_{1}$-th primitive root of unity. Moreover, for any $i_{1} \in\left\{0, \ldots, n_{1}-1\right\}$ and $i_{2} \in\left\{0, \ldots, n_{2}-1\right\}$, we have

$$
\begin{aligned}
\hat{a}_{i_{1} n_{2}+i_{2}} & =\sum_{0 \leqslant k_{1}<n_{1}} \sum_{0 \leqslant k_{2}<n_{2}} a_{k_{2} n_{1}+k_{1}} \omega^{\left(k_{2} n_{1}+k_{1}\right)\left(i_{1} n_{2}+i_{2}\right)} \\
& =\sum_{0 \leqslant k_{1}<n_{1}} \omega^{k_{1} i_{2}}\left(\sum_{0 \leqslant k_{2}<n_{2}} a_{k_{2} n_{1}+k_{1}}\left(\omega^{n_{1}}\right)^{k_{2} i_{2}}\right)\left(\omega^{n_{2}}\right)^{k_{1} i_{1}} .
\end{aligned}
$$

If $A_{1}$ and $A_{2}$ are algorithms for computing DFTs of length $n_{1}$ and $n_{2}$, we may use (1) to construct an algorithm for computing DFTs of length $n$ as follows. For each $k_{1} \in\{0, \ldots$, $\left.n_{1}-1\right\}$, the sum inside the brackets corresponds to the $i_{2}$-th coefficient of a DFT of the $n_{2}$-tuple $\left(a_{0 n_{1}+k_{1}}, \ldots, a_{\left(n_{2}-1\right) n_{1}+k_{1}}\right) \in \mathbb{F}_{q}^{n_{2}}$ with respect to $\omega^{n_{1}}$. Evaluating these inner DFTs requires $n_{1}$ calls to $A_{2}$. Next, we multiply by the twiddle factors $\omega^{k_{1} i_{2}}$, at a cost of $n$ operations in $\mathbb{F}_{q}$. Finally, for each $i_{2} \in\left\{0, \ldots, n_{2}-1\right\}$, the outer sum corresponds to the $i_{1}$-th coefficient of a DFT of an $n_{1}$-tuple in $\mathbb{F}_{q}^{n_{1}}$ with respect to $\omega^{n_{2}}$. These outer DFTs require $n_{2}$ calls to $A_{1}$. Iterating this decomposition for further factorizations of $n_{1}$ and $n_{2}$ yields the seminal Cooley-Tukey algorithm [2].

\section{Frobenius Fourier transforms}

Let $A$ be a polynomial in $\mathbb{F}_{q}[x]$ and let $\omega$ be a primitive root of unity in some extension $\mathbb{F}_{q^{\ell}}$ of $\mathbb{F}_{q}$. We write $\phi_{q}$ for the Frobenius map $a \mapsto a^{q}$ in $\mathbb{F}_{q}$ and notice that

$$
A\left(\phi_{q}(a)\right)=\phi_{q}(A(a))
$$

for any $a \in \mathbb{F}_{q^{\ell}}$. This formula implies many nontrivial relations for the DFT of $A$ : if $\omega^{i}=$ $\phi_{q}^{\circ k}\left(\omega^{j}\right)$, then we have $A\left(\omega^{i}\right)=\phi_{q}^{\circ k}\left(A\left(\omega^{j}\right)\right)$. In other words, some values of the DFT of $A$ can be deduced from others, and the advantage of the Frobenius transform introduced in [10] is to restrict the bulk of the evaluations to a minimum number of points. 
Let $n$ denote the order of the root $\omega$, and consider the set $\Omega=\left\{1, \omega, \omega^{2}, \ldots, \omega^{n-1}\right\}$. This set is clearly globally stable under $\phi_{q}$, so the group $\left\langle\phi_{q}\right\rangle$ generated by $\phi_{q}$ acts naturally on it. This action partitions $\Omega$ into disjoint orbits. Assume that we have a section $\Sigma$ of $\Omega$ that contains exactly one element in each orbit. Then formula (2) allows us to recover $\operatorname{DFT}_{\omega}(A)$ from the evaluations of $A$ at each of the points in $\Sigma$. The vector $(A(\sigma))_{\sigma \in \Sigma}$ is called the Frobenius DFT of $A$.

\section{FAST REDUCTION FROM $\mathbb{F}_{2}[x]$ TO $\mathbb{F}_{2^{60}}[x]$}

\subsection{Variant of the Frobenius DFT}

To efficiently reduce a multiplication in $\mathbb{F}_{2}[x]$ into DFTs over $\mathbb{F}_{2^{60}}$, we use an order $n$ that divides $2^{60}-1$ and such that $n=61 m$ for some integer $m$. We perform the decomposition (1) with $n_{1}=m$ and $n_{2}=61$. Let $\omega$ be a primitive $n$-th root of unity in $\mathbb{F}_{2^{60}}$. The discrete Fourier transform of $A \in \mathbb{F}_{2}[x]_{<n}$, given by $\left(A(1), A(\omega), A\left(\omega^{2}\right), \ldots, A\left(\omega^{n-1}\right)\right) \in$ $\mathbb{F}_{2^{60}}^{n}$, can be reorganized into 61 slices as follows

$$
\operatorname{DFT}_{\omega}(A)=\left(\left(A\left(\omega^{61 i}\right)\right)_{0 \leqslant i<m}\left(A\left(\omega^{61 i+1}\right)\right)_{0 \leqslant i<m}, \ldots,\left(A\left(\omega^{61 i+60}\right)\right)_{0 \leqslant i<m}\right) .
$$

The variant of the Frobenius DFT of $A$ that we introduce in the present paper corresponds to computing only the second slice:

$$
\begin{aligned}
E_{\omega:}: \mathbb{F}_{2}[x]_{<60 m} & \rightarrow \mathbb{F}_{2^{60}}^{m} \\
A & \mapsto\left(A\left(\omega^{61 i+1}\right)\right)_{0 \leqslant i<m} .
\end{aligned}
$$

Let us show that this transform is actually a bijection. The following lemma shows that the slices $\left(A\left(\omega^{61 i+2}\right)\right)_{0 \leqslant i<m}, \ldots,\left(A\left(\omega^{61 i+60}\right)\right)_{0 \leqslant i<m}$ can be deduced from the second slice $\left(A\left(\omega^{61 i+1}\right)\right)_{0 \leqslant i<m}$ using the action of the Frobenius map $\phi_{2}$.

LEMMA 1. Let $\Omega_{i}=\left\{\omega^{61 j+i}: 0 \leqslant j<m\right\}$ for $1 \leqslant i<61$. Then the action of $\left\langle\phi_{2}\right\rangle$ is transitive on the pairwise disjoint sets $\Omega_{1}, \ldots, \Omega_{60}$.

Proof. Let $1 \leqslant i<61$ and $0 \leqslant j<m$, we have $\phi_{2}\left(\omega^{61 j+i}\right)=\omega^{61 j^{\prime}+(2 i \bmod 61)}$ for some integer $0 \leqslant j^{\prime}<m$, so the action of $\left\langle\phi_{2}\right\rangle$ onto $\Omega_{1}, \ldots, \Omega_{60}$ is well defined. Notice that 2 is primitive for the multiplicative group $\mathbb{F}_{61}^{\times}$. This implies that for any $1 \leqslant i<61$ there exists $k$ such that $2^{k}=i \bmod 61$. Consequently we have $\phi_{2}^{\circ k}\left(\omega^{61 j+1}\right)=\omega^{61 j^{\prime}+i}$ for some $0 \leqslant j^{\prime}<m$, whence $\phi_{2}^{\circ k}\left(\Omega_{1}\right) \subseteq \Omega_{i}$. Since $\phi_{2}$ is injective the latter inclusion is an equality.

If we were needed the complete $\operatorname{DFT}_{\omega}(A)$, then we would still have to compute the first slice $\left(A\left(\omega^{61 i}\right)\right)_{0 \leqslant i<m}$. The second main new idea with respect to [10] is to discard this first slice and to restrict ourselves to input polynomials $A$ of degrees $<60 \mathrm{~m}$. In this way, $E_{\omega}$ can be inverted, as proved in the following proposition.

PROPOSITION 2. $E_{\omega}$ is bijective.

Proof. The dimensions of the source and destination spaces of $E_{\omega}$ over $\mathbb{F}_{2}$ being the same, it suffices to prove that $E_{\omega}$ is injective. Let $A \in \mathbb{F}_{2}[x]_{<60 m}$ be such that $E_{\omega}(A)=0$. By construction, $A$ vanishes at $m$ distinct values, namely $\omega^{61 i+1}$ for $0 \leqslant i<m$. Under the action of $\left\langle\phi_{2}\right\rangle$ it also vanishes at $60(m-1)$ other values by Lemma 1 , whence $A=0$. 


\subsection{Frobenius encoding}

We decompose the computation of $E_{\omega}$ into two routines. The first routine is written $F_{\omega}$ and called the Frobenius encoding:

$$
\begin{aligned}
F_{\omega}: \mathbb{F}_{2}[x]_{<60 m} & \rightarrow \mathbb{F}_{260}[x]_{<m} \\
A=\sum_{0 \leqslant k<60 m} a_{k} x^{k} & \mapsto \sum_{0 \leqslant k<m} \omega^{k}\left(\sum_{0 \leqslant l<60} a_{k+m l} \theta^{l}\right) x^{k} \text {, where } \theta=\omega^{m} .
\end{aligned}
$$

Below, we will choose $\theta$ in such a way that $F_{\omega}$ is essentially a simple reorganization of the coefficients of $A$.

We observe that the coefficients of $F_{\omega}(A)$ are part of the values of the inner DFTs of $A$ in the Cooley-Tukey formula (1), applied with $n_{1}=m$ and $n_{2}=61$. The second task is the computation of the corresponding outer DFT of order $m$ :

$$
\begin{aligned}
\operatorname{DFT}_{\tilde{\omega}}: \mathbb{F}_{2^{60}}[x]_{<m} & \rightarrow \mathbb{F}_{2^{60}}^{m} \\
\tilde{A} & \mapsto\left(\tilde{A}\left(\tilde{\omega}^{i}\right)\right)_{0 \leqslant i<m}, \text { where } \tilde{\omega}=\omega^{61} .
\end{aligned}
$$

PROPOSITION 3. $E_{\omega}=\operatorname{DFT}_{\tilde{\omega}} \circ F_{\omega}$.

Proof. This formula follows from (1):

$$
A\left(\omega^{61 i+1}\right)=\sum_{0 \leqslant k<m} \omega^{k}\left(\sum_{0 \leqslant l<61} a_{k+m l} \theta^{l}\right) \tilde{\omega}^{k i}=F_{\omega}(A)\left(\tilde{\omega}^{i}\right) .
$$

Summarizing, we have reduced the computation of a DFT of size $60 n / 61$ over $\mathbb{F}_{2}$ to a DFT of size $m=n / 61$ over $\mathbb{F}_{2^{60}}$. This reduction preserves data size.

\subsection{Direct transforms}

The computation of $F_{\omega}$ involves the evaluation of $m$ polynomials in $\mathbb{F}_{2}[x]_{<60}$ at $\theta=\omega^{m} \in \mathbb{F}_{2^{60}}$. In order to perform these evaluations fast, we fix the representation of $\mathbb{F}_{2^{60}}=\mathbb{F}_{2}[z] /(\mu(z))$ and the primitive root $\nu$ of unity of maximal order $2^{60}-1$ to be given by

$$
\begin{aligned}
\mu(z) & =\left(z^{61}-1\right) /(z-1) \\
v & =z^{18}+z^{6}+1 \bmod \mu(z) .
\end{aligned}
$$

Setting $\omega=v^{\left(2^{60}-1\right) / n}$ and $\theta=v^{\left(2^{60}-1\right) / 61}$, it can be checked that $\theta=z \bmod \mu(z)$. Evaluation of a polynomial in $\mathbb{F}_{2}[x]_{<60}$ at $\theta$ can now be done efficiently.

\section{Algorithm 1}

Input: $A(x)=\sum_{0 \leqslant i<60 m} a_{i} x^{i}$.

Output: $F_{\omega}(A)$.

Assumption: $n=61 m$ divides $2^{60}-1$.

1. For $i=0, \ldots, m-1$, build $P_{i}(z)=\sum_{0 \leqslant j<60} a_{i+m j} z^{j} \bmod \mu(z) \in \mathbb{F}_{2^{60}}$.

2. Return $P_{0}+\omega P_{1} x+\omega^{2} P_{2} x^{2}+\cdots+\omega^{m-1} P_{m-1} x^{m-1}$.

PROPOSITION 4. Algorithm 1 is correct. 
Proof. This deduces immediately from the definition of $F_{\omega}$ in formula (3), using the fact that $\theta=z \bmod \mu(z)$ in our representation.

\section{Algorithm 2}

Input: $A \in \mathbb{F}_{2}[x]_{<60 m}$.

Output: $E_{\omega}(A)$.

Assumption: $n=61 m$ divides $2^{60}-1$.

1. Compute the Frobenius encoding $\tilde{A}(x) \in \mathbb{F}_{2^{60}}[x]_{<m}$ of $A$ by Algorithm 1 .

2. Compute the DFT of $\tilde{A}$ with respect to $\tilde{\omega}$.

PROPOSITION 5. Algorithm 2 is correct.

Proof. The correctness simply follows from Propositions 3 and 4.

\subsection{Inverse transforms}

By combining Propositions 2 and 3, the map $F_{\omega}$ is invertible and its inverse may be computed by the following algorithm.

\section{Algorithm 3}

Input: $\tilde{A}(x)=\sum_{i \geqslant 0} \tilde{a}_{i} x^{i} \in \mathbb{F}_{2^{60}}[x]_{<m}$.

Output: $F_{\omega}^{-1}(\tilde{A})$.

Assumption: $n=61 m$ divides $2^{60}-1$.

1. For $i=0, \ldots, m-1$, build the preimage $P_{i}(z):=\sum_{0 \leqslant j<60} p_{i, j} z^{j}$ of $\omega^{-i} \tilde{a}_{i}$.

2. Return $\sum_{0 \leqslant i<m} \sum_{0 \leqslant j<60} p_{i, j} x^{i+m j}$.

PROPOSITION 6. Algorithm 3 is correct.

Proof. This is a straightforward inversion of Algorithm 1.

\section{Algorithm 4}

Input: $\hat{a} \in \mathbb{F}_{2^{60}}^{m}$.

Output: $E_{\omega}^{-1}(\hat{a})$.

Assumption: $n=61 \mathrm{~m}$ divides $2^{60}-1$.

1. Compute the inverse DFT $\tilde{A} \in \mathbb{F}_{2^{60}}[x]_{<m}$ of $\hat{a}$ with respect to $\tilde{\omega}$.

2. Compute the Frobenius decoding $A$ of $\tilde{A}$ by Algorithm 3 and return $A$.

PROPOSITION 7. Algorithm 4 is correct.

Proof. The correctness simply follows from Propositions 3 and 6.

\subsection{Multiplication in $\mathbb{F}_{2}[x]$}

Using the standard technique of multiplication by evaluation-interpolation, we may now compute products in $\mathbb{F}_{2}[x]$ as follows: 


\section{Algorithm 5 \\ Input: $A, B \in \mathbb{F}_{2}[x]_{<\ell}$. \\ Output: $A B$}

1. Let $m \geqslant(2 \ell-1) / 60$ be such that $n=61 m$ divides $2^{60}-1$.

2. Let $\omega=v^{\left(2^{60}-1\right) / n}$ be the privileged root of unity of order $n$.

3. Compute $E_{\omega}(A)$ and $E_{\omega}(B)$ by Algorithm 2.

4. Compute $\hat{c}$ as the entry-wise product of $E_{\omega}(A)$ and $E_{\omega}(B)$.

5. Compute $C(x)=E_{\omega}^{-1}(\hat{c})$ by Algorithm 4 and return $C$.

PROPOSITION 8. Algorithm 4 is correct.

Proof. The correctness simply follows from Propositions 5 and 7 and using the fact that $E_{\omega}(A B)=E_{\omega}(A) E_{\omega}(B)$, since $m \geqslant(2 \ell-1) / 60$.

For step 1, the actual determination of $m$ has been discussed in [6, section 3]. In fact it is often better not to pick the smallest possible value for $m$ but a slightly larger one that is also very smooth. Since $2^{60}-1$ admits many small prime divisors, such smooth values of $m$ usually indeed exist.

\section{IMPLEMENTATION DETAILS}

We follow INTEL's terminology and use the term quad word to denote a unit of 64 bits of data. In the rest of the paper we use the C99 standard for presenting our source code. In particular a quad word representing an unsigned integer is considered of type uint64_t.

Our implementations are done for an AVX2-enabled processor and an operating system compliant to System V Application Binary Interface. The C++ library NUMERIX of MATHEMAGIX [12] (http: //www . mathemagix org) defines wrappers for AVX types. In particular, avx_uint64_t represents an SIMD vector of 4 elements of type uint64_t. Recall that the platform disposes of 16 AVX registers which must be allocated accurately in order to minimize read and write accesses to the memory.

Our new polynomial product is implemented in the JUSTINLINE library of MATHEMAGIX. The source code is freely available from revision 10681 of our SVN server (https://gforge.inria.fr/projects/mmx/). Main sources are in justinline/ src/frobenius_encode_f2_60.cpp for the Frobenius encoding and in justinline/ $\mathrm{mmx}$ /polynomial_f2_amd64_avx2_clmul.mmx for the top level functions. Related test and bench files are also available from dedicated directories of the JUSTINLINE library. Let us further mention here that our MATHEMAGIX functions may be easily exported to $\mathrm{C}++[11]$.

\subsection{Packed representations}

Polynomials over $\mathbb{F}_{2}$ are supposed to be given in packed representation, which means that coefficients are stored as a vector of contiguous bits in memory. For the implementation considered in this paper, a polynomial of degree $\ell-1$ is stored into $\lceil\ell / 64\rceil$ quad words, starting with the low-degree coefficients: the constant term is the least significant bit of the first word. The last word is suitably padded with zeros. 
Reading or writing one coefficient or a range of coefficients of a polynomial in packed representation must be done carefully to avoid invalid memory access. Let $A$ be such a polynomial of type uint64_t*. Reading the coefficient $a_{i}$ of degree $i$ in $A$ is obtained as $(A[i>6]>(i \&$ 63) $)$ \& 1. However, reading or writing a single coefficient should be avoided as much as possible for efficiency, so we prefer handling ranges of 256 bits. In the sequel the function of prototype

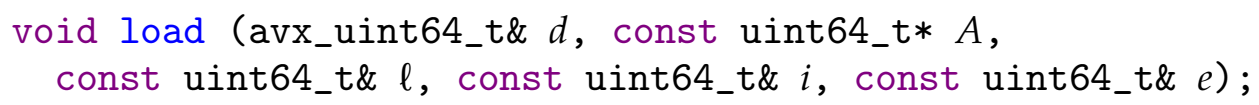

returns the $e \leqslant 256$ bits of $A$ starting from $i$ into $d$. Bits beyond position $\ell$ are considered to be zero. The converse operation saves the $e \leqslant 256$ first bits of $s$ into $A$ at position starting from the $i$-th bit; it has prototype

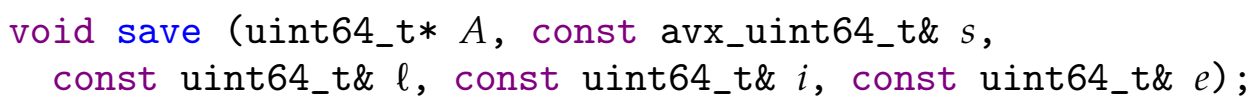

For arithmetic operations in $\mathbb{F}_{2^{60}}$ we refer the reader to [6, section 3.1]. In the sequel we only appeal to the function

uint64_t f2_60_mul (const uint64_t\& $a$, const uint64_t\& $b$ );

that multiplies the two elements $a$ and $b$ of $\mathbb{F}_{2^{60}}$ in packed representation.

We also use a packed column-major representation for matrices over $\mathbb{F}_{2}$. For instance, an $8 \times 8$ bit matrix $\left(M_{i, j}\right)_{0 \leqslant i<8,0 \leqslant j<8}$ is encoded as a quad word whose $(8 j+i)$-th bit is $M_{i, j}$. Similarly, a $256 \times \ell$ matrix $\left(M_{i, j}\right)_{0 \leqslant i<256,0 \leqslant j<\ell}$ may be seen as a vector $v$ of type avx_uint64_t*, so $M_{i, j}$ corresponds to the $i$-th bit of $v[j]$.

\subsection{Matrix transposition}

The Frobenius encoding essentially boils down to matrix transpositions. Our main building block is $256 \times 64$ bit matrix transposition. We decompose this transposition in a suitable way with regards to data locality, register allocation and vectorization.

For the computation of general transpositions, we repeatedly make use of the wellknown divide and conquer strategy: to transpose an $n \times \ell$ matrix $M$, where $n$ and $\ell$ are even, we decompose $M=\left(\begin{array}{ll}A & B \\ C & D\end{array}\right)$, where $A, B, C, D$ are $n / 2 \times \ell / 2$ matrices; we swap the anti-diagonal blocks $B$ and $C$ and recursively transpose each block $A, B, C, D$.

\subsubsection{Transposing packed $8 \times 8$ bit matrices}

The basic task we begin with is the transposition of a packed $8 \times 8$ bit matrix. The solution used here is borrowed from [17, Chapter 7 , section 3].

\section{Function 1}

Input: $\left(M_{i, j}\right)_{0 \leqslant i<8,0 \leqslant j<8}$ in packed representation.

Output: The transpose $\left(N_{i, j}\right)_{0 \leqslant i<8,0 \leqslant j<8}$ of $M$ in packed representation. 
uint64_t

packed_matrix_bit_8x8_transpose (const uint64_t\& $M$ ) \{

1. uint64_t $N=M$;

2. static const uint64_t mask_4 $=0 \times 00000000 \mathrm{f} 0 \mathrm{f} 0 \mathrm{f} 0 \mathrm{f} 0$;

3. static const uint64_t mask_2 $=0 \times 0000$ cccc0000cccc;

4. static const uint64_t mask_1 $=0 \mathrm{x} 00 \mathrm{aa} 00 \mathrm{aa00aa00aa;}$

5. uint64_t $a$;

6. $a=((N>>28)-N)$ \& $\operatorname{mask}_{-} 4 ; N=N-a$;

7. $a=a<<28 ; N=N-a$;

8. $a=((N>14)-N)$ \& mask_2; $N=N$ - $a$;

9. $a=a<<14 ; N=N-a$;

10. $a=((N>>7)-N) \& \operatorname{mask}_{-} 1 ; N=N-a$;

11. $a=a<<7 ; \quad N=N-a$;

12. return $N ;\}$

In steps 6 and 7, the anti-diagonal $4 \times 4$ blocks are swapped. In steps 8 and 9 , the matrix $N$ is seen as four $4 \times 4$ matrices whose anti-diagonal $2 \times 2$ blocks are swapped. In steps 10 and 11, the matrix $N$ is seen as sixteen $2 \times 2$ matrices whose anti-diagonal elements are swapped. All in all, 18 instructions, 3 constants and one auxiliary variable are needed to transpose a packed $8 \times 8$ bit matrix in this way.

One advantage of the above algorithm is that it admits a straightforward AVX vectorization that we will denote by

avx_uint64_t

avx_packed_matrix_bit_8x8_transpose (const avx_uint64_t\& $M$ );

This routine transposes four $8 \times 8$ bit matrices $M_{0}, M_{1}, M_{2}, M_{3}$ that are packed successively into an AVX register of type avx_uint64_t. We emphasize that this task is not the same as transposing a $32 \times 8$ or $8 \times 32$ bit matrices.

Remark 9. The BMI2 technology gives another method for transposing $8 \times 8$ bit matrices:

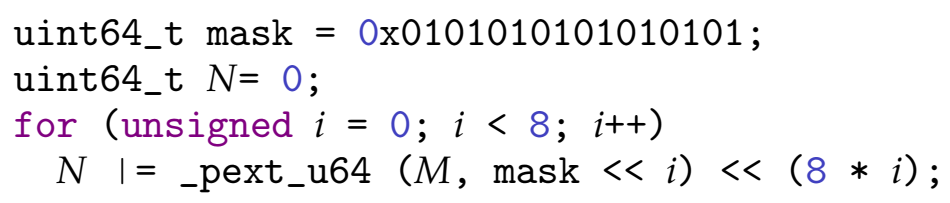

The loop can be unrolled while precompting the shift amounts and masks, which leads to a faster sequential implementation. Unfortunately this approach cannot be vectorized with the AVX2 technology. Other sequential solutions even exist, based on lookup tables or integer arithmetic, but their vectorization is again problematic. Practical efficiencies are reported in section 5 .

\subsubsection{Transposing four $8 \times 8$ byte matrices simultaneously}

Our next task is to design a transposition algorithm of four packed $8 \times 8$ byte matrices simultaneously. More precisely, it performs the following operation on a packed $32 \times 8$ byte matrix:

$$
\left(\begin{array}{l}
M_{0} \\
M_{1} \\
M_{2} \\
M_{3}
\end{array}\right) \rightarrow\left(\begin{array}{l}
M_{0}^{\top} \\
M_{1}^{\top} \\
M_{2}^{\top} \\
M_{3}^{\top}
\end{array}\right)
$$


where the $M_{i}$ are $8 \times 8$ blocks. This operation has the following prototype in the sequel:

void avx_packed_matrix_byte_8x8_transpose

(avx_uint64_t* dest, const avx_uint64_t* src);

This function works as follows. First the input src is loaded into eight AVX registers $r_{0}, \ldots, r_{7}$. Each $r_{i}$ is seen as a vector of four uint64_t: for $j \in\{0, \ldots, 3\}, r_{0}[j], \ldots, r_{7}[j]$ thus represent the $8 \times 8$ byte matrix $M_{j}$. Then we transpose these four matrices simultaneously in-register by means of AVX shift and blend operations over 32, 16 and 8 bits entries in the spirit of the aforementioned divide and conquer strategy.

\subsubsection{Transposing $256 \times 64$ bit matrices}

Having the above subroutines at our disposal, we can now present our algorithm to transpose a packed $256 \times 64$ bit matrix. The input bit matrix of type avx_int64_t* is written $\left(M_{i, j}\right)_{0 \leqslant i<256,0 \leqslant j<64}$. The transposed output matrix is written $\left(N_{i, j}\right)_{0 \leqslant i<64,0 \leqslant j<256}$ and has type uint64_t*. We first compute the auxiliary byte matrix $T$ as follows:

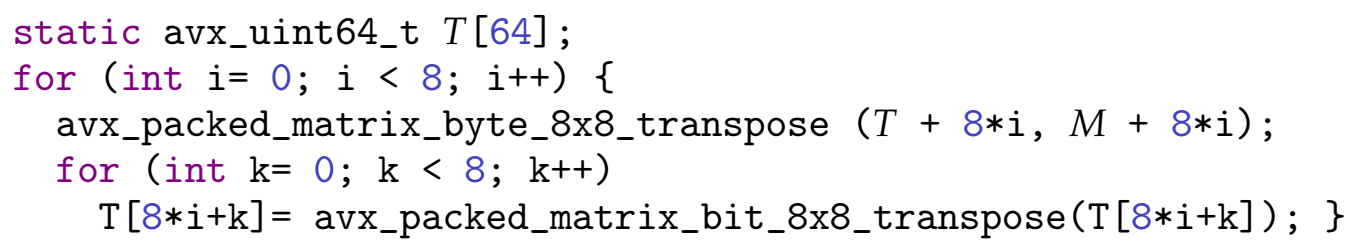

If we write $M_{i, k: l}$ for the byte representing the packed bit vector $\left(M_{i, k}, \ldots, M_{i, l}\right)$, then $T$ contains the following $32 \times 64$ byte matrix:

$$
\left(\begin{array}{ccc|ccc|c|ccc}
M_{0,0: 7} & \ldots & M_{56,0: 7} & M_{0,8: 15} & \ldots & M_{56,8: 15} & \ldots & M_{0,56: 63} & \ldots & M_{56,56: 63} \\
\vdots & & \vdots & \vdots & & \vdots & & \vdots & & \vdots \\
M_{7,0: 7} & \ldots & M_{63,0: 7} & M_{7,8: 15} & \ldots & M_{63,8: 15} & \ldots & M_{7,56: 63} & \ldots & M_{63,56: 63} \\
\hline M_{64,0: 7} & \ldots & M_{120,0: 7} & M_{64,8: 15} & \ldots & M_{120,8: 15} & \ldots & M_{64,56: 63} & \ldots & M_{120,56: 63} \\
\vdots & & \vdots & \vdots & & \vdots & & \vdots & & \vdots \\
M_{71,0: 7} & \ldots & M_{127,0: 7} & M_{71,8: 15} & \ldots & M_{127,8: 15} & \ldots & M_{71,56: 63} & \ldots & M_{127,56: 63} \\
\hline M_{128,0: 7} & \ldots & M_{184,0: 7} & M_{128,8: 15} & \ldots & M_{184,8: 15} & \ldots & M_{128,56: 63} & \ldots & M_{184,56: 63} \\
\vdots & & \vdots & \vdots & & \vdots & & \vdots & & \vdots \\
M_{135,0: 7} & \ldots & M_{191,0: 7} & M_{135,8: 15} & \ldots & M_{191,8: 15} & \ldots & M_{135,56: 63} & \ldots & M_{191,56: 63} \\
\hline M_{192,0: 7} & \ldots & M_{248,0: 7} & M_{192,8: 15} & \ldots & M_{248,8: 15} & \ldots & M_{192,56: 63} & \ldots & M_{248,56: 63} \\
\vdots & & \vdots & \vdots & & \vdots & & \vdots & & \vdots \\
M_{199,0: 7} & \ldots & M_{255,0: 7} & M_{199,8: 15} & \ldots & M_{255,8: 15} & \ldots & M_{199,56: 63} & \ldots & M_{255,56: 63}
\end{array}\right) .
$$

First, for all $0 \leqslant i \leqslant 7$, we load column $8 i$ into the AVX register $r_{i}$. We interpret these registers as forming a $32 \times 8$ byte matrix that we transpose in-registers. This transposition is again performed in the spirit of the aforementioned divide and conquer strategy and makes use of various specific AVX2 instructions. We obtain

$$
\left(\begin{array}{cccc|cccc|c}
M_{0,0: 7} & M_{1,0: 7} & \ldots & M_{7,0: 7} & M_{64,0: 7} & M_{65,0: 7} & \ldots & M_{71,0: 7} & \ldots \\
M_{0,8: 15} & M_{1,8: 15} & \ldots & M_{7,8: 15} & M_{64,8: 15} & M_{65,8: 15} & \ldots & M_{71,8: 15} & \ldots \\
\vdots & \vdots & & \vdots & \vdots & \vdots & & \vdots & \\
M_{0,56: 63} & M_{1,56: 63} & \ldots & M_{7,56: 63} & M_{64,56: 63} & M_{65,56: 63} & \ldots & M_{71,56: 63} & \ldots
\end{array}\right)
$$

More precisely, for $i=0, \ldots, 7$, the group of four consecutive columns from $4 i$ until $4 i+3$ is in the register $r_{i}$. We save the registers $r_{0}, \ldots, r_{7}$ at the addresses $N, N+4, N+64, N+68$, $N+128, N+132, N+192$ and $N+196$. 
For each $k=1, \ldots, 7$, we build a similar $32 \times 8$ byte matrix from the columns $k, 8+k, \ldots$, $56+k$ of $T$, and transpose this matrix using the same algorithm. This time the result is saved at the addresses $N^{\prime}, N^{\prime}+4, N^{\prime}+64, N^{\prime}+68, N^{\prime}+128, N^{\prime}+132, N^{\prime}+192$ and $N^{\prime}+196$, where $N^{\prime}=N+8 k$. This yields an efficient routine for transposing $M$ into $N$, whose prototype is given by

void packed_matrix_bit_256x64_transpose

(uint64_t* $N$, (const avx_uint64_t*) $M$ );

\subsection{Frobenius encoding}

If the input polynomial $A$ has degree less than $\ell \leqslant 60 \mathrm{~m}$ and is in packed representation, then it can also be seen as a $m \times 60$ matrix in packed representation (except a padding with zeros could be necessary to adjust the size).

In this setting, the polynomials $P_{i}$ of Algorithm 1 are simply read as the rows of the matrix. Therefore, to compute the Frobenius encoding $F_{\omega}(A)$, we only need to transpose this matrix, then add 4 rows of zeros for alignment (because we store one element of $\mathbb{F}_{2^{60}}$ per quad word) and multiply by twiddle factors. This leads to the following implementation:

\section{Function 2}

Input: $A(x)=\sum_{0 \leqslant i<\ell} a_{i} x^{i} \in \mathbb{F}_{2}[x]$.

Output: $F_{\omega}(A)$ stored from pointer $d$ to $m$ allocated quad words.

Assumptions: $n=61 \mathrm{~m}$ divides $2^{60}-1$ and $\ell \leqslant 60 \mathrm{~m}$.

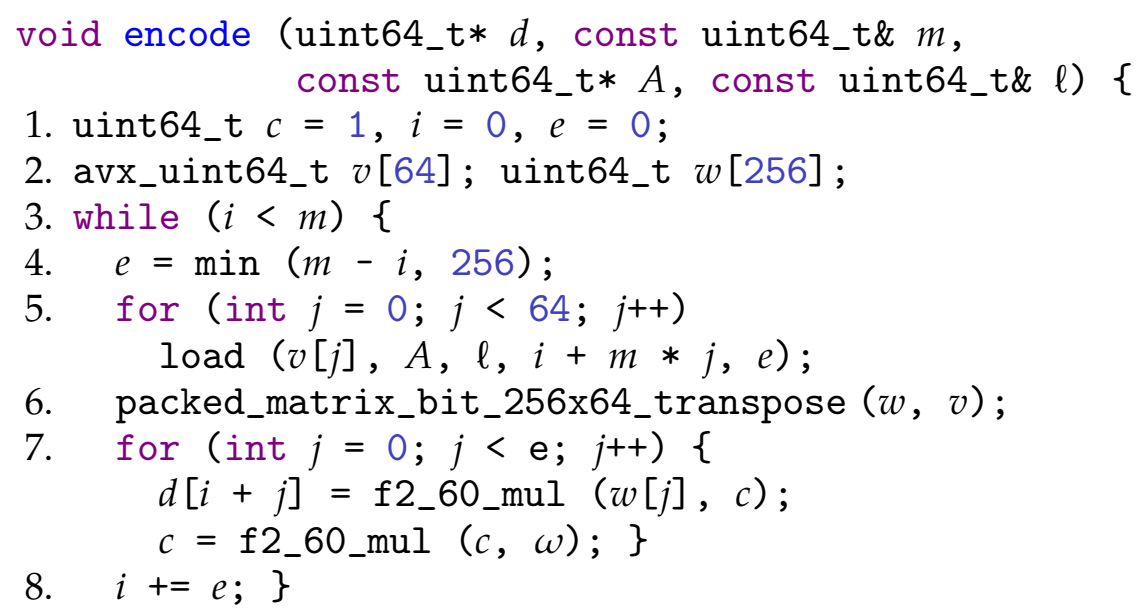

Remark. To optimize read accesses, it is better to run loop 5 for $j<\lceil l / m\rceil$ and to initialize the remaining $v[j]$ to zero. Indeed, for a product of degree $d$, we typically multiply two polynomials of degree $\simeq d / 2$, which means $\ell<30 \mathrm{~m}$ when computing the direct transform.

The Frobenius decoding consists in inverting the encoding. The implementation issues are the same, so we refer to our source code for further details.

\section{TIMINGS}

The platform considered in this paper is equipped with an INTEL(R) CORE(TM) i7-6700 CPU at $3.40 \mathrm{GHz}$ and $32 \mathrm{~GB}$ of $2133 \mathrm{MHz}$ DDR4 memory. This CPU features AVX2, BMI2 and CLMUL technologies (family number 6 and model number 94). The platform runs the STRETCH GNU DEBIAN operating system with a 64 bit LINUX kernel version 4.3. We compile with GCC [5] version 5.4. 


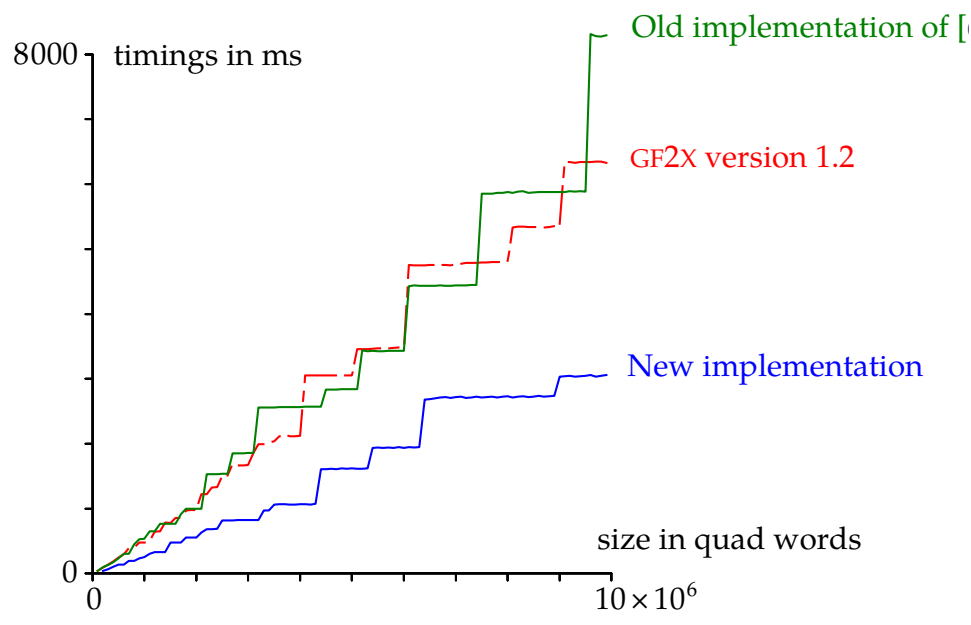

Figure 1. Products in $\mathbb{F}_{2}[x]_{<\ell}$, input size $\lceil\ell / 64\rceil$ quad words, timings in milliseconds.

We use version 1.2 of the GF2x library (https://gforge.inria.fr/projects/ $\mathrm{gf} 2 \mathrm{x} /$, released in July 2017) - it makes use of the CLMUL features of the platform. We tuned it to our platform during the installation process up to 32000000 input quad words.

\section{Frobenius encoding}

Concerning the cost of the Frobenius encoding and decoding, Function 1 takes about 20 CPU cycles when compiled with the sole -03 option. With the additional options -mtune=native -mavx2 -mbmi2, the BMI2 version of Remark 9 takes about 16 CPU cycles. The vectorized version of Function 1 transposes four packed $8 \times 8$ bit matrices simultaneously in about 20 cycles, which makes an average of 5 cycles per matrix.

It it interesting to examine the performance of the sole transpositions made during the Frobenius encoding and decoding (that is discarding products by twiddle factors in $\mathbb{F}_{2^{60}}$. From sizes of a few kilobytes this average cost per quad word is about 8 cycles with the AVX2 technology, and it is about 23 cycles without. Unfortunately the vectorization speed-up is not as close to 4 as we would have liked.

Since the encoding and decoding costs are linear, their relative contribution to the total computation time of polynomial products decreases for large sizes. For two input polynomials in $\mathbb{F}_{2}[x]$ of $2^{16}$ quad words, the contribution is about $15 \%$; for $2^{22}$ quad words, it is about $10 \%$.

\section{Polynomial product}

In Figure 1 we report timings in milliseconds for multiplying two polynomials in $\mathbb{F}_{2}[x]_{<\ell}$, hence each of input size $\lceil\ell / 64\rceil$ quad words-indicated in abscissa and obtained from justinline/bench/polynomial_f2_bench.mmx. Notice that our implementation in [6] was faster than version 1.1 of GF2X, but is now of similar speed as version 1.2. The speed-up between our old and new implementations is not far from the factor 2 predicted by the asymptotic complexity analysis. Let us mention that our implementation becomes faster than GF2X when $\lceil\ell / 64\rceil$ is larger than 2048. 


\begin{tabular}{|l|c|c|r|r|c|c|}
\hline \multicolumn{1}{|c|}{$r$} & 1 & 2 & \multicolumn{1}{c|}{4} & \multicolumn{1}{c|}{8} & 16 & 32 \\
\hline this paper & 12 & 51 & 212 & 896 & 3969 & 18953 \\
\hline GF2X & 22 & 182 & 1457 & 11856 & 92858 & 745586 \\
\hline
\end{tabular}

Table 1. Products of $r \times r$ matrices over $\mathbb{F}_{2}[x]$, for degree $64 \cdot 2^{16}$, in milliseconds.

\section{Polynomial matrix product}

As in [6], one major advantage of DFTs over the Babylonian field $\mathbb{F}_{2^{60}}$ is the compactness of the evaluated FFT-representation of polynomials. This makes linear algebra over $\mathbb{F}_{2}[x]$ particularly efficient: instead of multiplying $r \times r$ matrices over $\mathbb{F}_{2}[x]_{<\ell}$ naively by means of $r^{3}$ polynomial products of degree $<\ell$, we use the standard evaluation-interpolation approach. In our context, this comes down to: (a) computing the $2 r^{2}$ Frobenius encodings, (b) the $2 r^{2}$ direct DFTs of all entries of the two matrices to be multiplied, (c) performing the $\approx 2 \ell / 60$ products of $r \times r$ matrices over $\mathbb{F}_{2^{60}},(\mathrm{~d})$ computing the $r^{2}$ inverse DFTs and Frobenius decodings of the so-computed matrix products.

Timings for matrices over $\mathbb{F}_{2}[x]$ are obatined from justinline/bench/ matrix_polynomial_f2_bench.mmx and are reported in Table 1. The row "this paper" confirms the practical gain of this fast approach within our implementation. For comparison, the row "GF2X" shows the cost of computing the product naively, by doing $r^{3}$ polynomial multiplications using GF2x. More efficient evaluation-interpolation based approaches [9, Section 2] for matrix multiplication can in principle be combined with Schönhage's triadic polynomial multiplication [15] as implemented in GF2X. However, this would require an additional implementation effort and also lead to an extra constant overhead with respect to our approach.

\section{CONCLUSION}

The present paper describes a major new approach for the efficient computation of large carryless products. It confirms the excellent arithmetic properties of the Babylonian field $\mathbb{F}_{2^{60}}$ for practical purposes, when compared to the fastest previously available strategies.

Improvements are still possible for our implementation of DFTs over $\mathbb{F}_{2^{60}}$. First, taking advantage of the more recent AVX-512 technologies is an important challenge. This is difficult due to the current lack of 256 or 512 bit SIMD counterparts for the vpclmulqdq assembly instruction (carryless multiplication of two quad words). However, larger vector instruction would be beneficial for matrix transposition, and even more taking into account that there are twice as many 512 bit registers as 256 bit registers; so we can expect a significant speed-up for the Frobenius encoding/decoding stages. The second expected improvement concerns the use of truncated Fourier transforms $[8,13]$ in order to smoothen the graph from Figure 1. Finally we expect that our new ideas around the Frobenius transform might be applicable to other small finite fields.

\section{BIBLIOGRAPHY}

[1] R. P. Brent, P. Gaudry, E. Thomé, and P. Zimmermann. Faster multiplication in GF(2) [ $x$ ]. In A. van der Poorten and A. Stein, editors, Algorithmic Number Theory, volume 5011 of Lect. Notes Comput. Sci., pages 153-166. Springer Berlin Heidelberg, 2008. 
[2] J. W. Cooley and J. W. Tukey. An algorithm for the machine calculation of complex Fourier series. Math. Computat., 19:297-301, 1965.

[3] S. Gao and T. Mateer. Additive fast Fourier transforms over finite fields. IEEE Trans. Inform. Theory, 56(12):6265-6272, 2010.

[4] J. von zur Gathen and J. Gerhard. Modern Computer Algebra. Cambridge University Press, 3rd edition, 2013.

[5] GCC, the GNU Compiler Collection. Software available at http: //gcc . gnu . org, from 1987.

[6] D. Harvey, J. van der Hoeven, and G. Lecerf. Fast polynomial multiplication over $F_{2^{60}}$ In M. Rosenkranz, editor, Proceedings of the ACM on International Symposium on Symbolic and Algebraic Computation, ISSAC '16, pages 255-262. ACM, 2016.

[7] D. Harvey, J. van der Hoeven, and G. Lecerf. Faster polynomial multiplication over finite fields. J. ACM, 63(6), 2017. Article 52.

[8] J. van der Hoeven. The truncated Fourier transform and applications. In J. Schicho, editor, Proceedings of the 2004 International Symposium on Symbolic and Algebraic Computation, ISSAC '04, pages 290-296. ACM, 2004.

[9] J. van der Hoeven. Newton's method and FFT trading. J. Symbolic Comput., 45(8):857-878, 2010.

[10] J. van der Hoeven and R. Larrieu. The Frobenius FFT. In M. Burr, editor, Proceedings of the 2017 ACM on International Symposium on Symbolic and Algebraic Computation, ISSAC '17, pages 437-444. ACM, 2017.

[11] J. van der Hoeven and G. Lecerf. Interfacing Mathemagix with $\mathrm{C}++$. In M. Monagan, G. Cooperman, and M. Giesbrecht, editors, Proceedings of the 2013 ACM on International Symposium on Symbolic and Algebraic Computation, ISSAC '13, pages 363-370. ACM, 2013.

[12] J. van der Hoeven and G. Lecerf. Mathemagix User Guide. https : //hal . archives-ouvertes.fr/ hal-00785549, 2013.

[13] R. Larrieu. The truncated Fourier transform for mixed radices. In M. Burr, editor, Proceedings of the 2017 ACM on International Symposium on Symbolic and Algebraic Computation, ISSAC '17, pages 261-268. ACM, 2017.

[14] Sian-Jheng Lin, Wei-Ho Chung, and S. Yunghsiang Han. Novel polynomial basis and its application to Reed-Solomon erasure codes. In 2014 IEEE 55th Annual Symposium on Foundations of Computer Science (FOCS), pages 316-325. IEEE, 2014.

[15] A. Schönhage. Schnelle Multiplikation von Polynomen über Körpern der Charakteristik 2. Acta Infor., 7:395-398, 1977.

[16] A. Schönhage and V. Strassen. Schnelle Multiplikation großer Zahlen. Computing, 7:281-292, 1971.

[17] H. S. Warren. Hacker's Delight. Addison-Wesley, 2nd edition, 2012. 